\title{
RELACIÓN ENTRE LA LONGITUD RELATIVA DE PIERNAS CORTAS CON SÍNDROME METABÓLICO Y SUS COMPONENTES EN ADULTOS JÓVENES DE OAXACA.
}

\author{
EVALUATION OF SHORT RELATIVE LENGTH LEG WITH METABOLIC SYNDROME AND ITS COMPONENTS IN \\ YOUNG ADULTS OF OAXACA
}

\author{
Montes de Oca-Juárez Olaf*, Cruz-Gaspar Anahí*, López-Toledo Sabina*. \\ * Universidad de la Sierra Sur (UNSIS), Oaxaca. México.
}

\section{RESUMEN}

Introducción. La longitud relativa de pierna (LRP) ha retomado importancia, no solo como indicador de crecimiento, sino por la posible asociación que tienen las piernas cortas y estaturas bajas con la presencia de síndrome metabólico (SM). Objetivo: Evaluar relación entre la longitud relativa de piernas cortas y el síndrome metabólico y sus componentes en adultos jóvenes de Oaxaca. Material y Método: Se realizó un estudio transversal en una muestra de 224 estudiantes entre 18 a 30 años. Se evaluó LRP y para diagnosticar SM se hicieron mediciones antropométricas y bioquímicas acordes al Adult Treatment Panel III (ATP-III). Para el análisis se eligieron grupos extremos (piernas cortas y largas) del cual se realizó el análisis de comparaciones múltiples de medias con cada uno de los componentes clínicos, bioquímicos y antropométricos del SM, y mediante regresión logística binaria se determinó significancia estadística. Resultados: Se determinó que el 10.7\% presentaba piernas cortas con una prevalencia de SM en un 32.5\%. No se encontró asociación de la LRP corta con el SM o alguno de los componentes del ATP III. Conclusiones: Se determinó que la presencia de piernas cortas en la población evaluada no se relaciona con SM ni las piernas largas con su prevención.

Palabras Clave: Longitud relativa de pierna, síndrome metabólico, obesidad.

\section{ABSTRACT}

Introduction: Assessment of relative leg length (RLL) has become important, not only as a growth indicator, but also because of the possible association of short legs and stature with the presence of metabolic syndrome (MS) or one of its components. Objective: Determine association between delay growth and metabolic syndrome, as well as its components in young adults in Oaxaca. Material and method: Cross-sectional study was conducted in a sample of 224 students between 18-30 years. RLL, anthropometrics and laboratory testing were measured to make the diagnosis for MS according to Adult Treatment Panel III (ATP-III). For the analysis, extreme groups were chosen (short and long legs) from which the analysis of multiple comparisons of means with each of the clinical, biochemical and anthropometric components of MS was performed, and statistical significance was determined by means of binary logistic regression. Results: It was determined that $10.7 \%$ had short legs with a prevalence of MS of $32.5 \%$. No association of short LRP with SM or any of the ATP III components was found. Conclusions: It was determined that the presence of short legs in this population is not related to MS and long legs with its prevention.

Key words: Relative leg length, metabolic syndrome, obesity.

Correspondencia: Olaf Montes de Oca Juárez licnut.olaf@gmail.com

Recibido: 10 de febrero 2021, aceptado: 26 de mayo 2021

(C) Autor2021

\section{(c) (i)}

DOI: https://doi.org/10.29105/respyn20.3-3

Citation: Montes de Oca-Juárez O., Cruz-Gaspar A., López-Toledo S. (2021) Evaluación de la relación entre la longitud relativa de piernas cortas con síndrome metabólico y sus componentes en adultos jóvenes de Oaxaca. Revista Salud Pública y Nutrición, 20 (3), 18-25. 


\section{Artículo Original}

\section{Introducción}

El crecimiento y la proporción corporal se han considerado recientemente como posibles factores de riesgo vinculados al síndrome metabólico (SM). Los mecanismos para explicar esta relación no han sido del todo esclarecidos, sin embargo, se centran en la teoría del genotipo ahorrador, donde el organismo de individuos cuyo ambiente sea precario y adverso favorecerá el cumplimiento de funciones vitales, mientras que aquellas secundarias como el crecimiento se verán descompensadas; de igual manera afectando a la distribución corporal que teóricamente es equitativa (50\% para segmentos superiores y 50\% para segmentos inferiores) (Bogin, Varela y Ríos, 2010). Cuando se superan los factores ambientales adversos que impedían a los sujetos crecer, su organismo tratará de compensar y ahorrar energía para evitar situaciones similares (Lawlor, Taylor, Davey, Gunnell y Ebrahim, 2004). Esto queda respaldado en estudios longitudinales de pacientes con antecedentes de desnutrición en la infancia, donde en la adultez presentan obesidad o son más propensos a ella (Fischer y Galler, 2015; Sawaya, Martins, Hoffman y Roberts, 2003; Sawaya y Roberts, 2003). Además de esta situación de ahorro de energía a expensas del tejido adiposo, también se ha propuesto que estos mismos sujetos descompensan el crecimiento y maduración de órganos, de los cuales uno de los más estudiados es el páncreas, donde se ha visto una reducción en el tamaño final, comprometiendo su función y producción de las células $\beta$, haciéndolos susceptibles a diabetes (Hales y Barker, 2013).

Este estudio evalúa la proporción corporal a partir de la medición de la longitud relativa de pierna, que se calcula mediante la diferencia entre la medición de estatura de pie y talla sentado, esta última se mide desde el vértice de la cabeza hasta los glúteos sentados (Mueller y Pereira, 2015). Y tiene como objetivo evaluar la relación de la longitud relativa de piernas cortas con el SM en una población de estudiantes universitarios de la Sierra Sur de Oaxaca. Considerandose de relevancia ante el crecimiento alarmante de SM en el país y en población adolescente cuya prevalencia se calcula en $27.9 \%$ (Aguilar-Salinas y Rojas, 2012) además que en México hay pocos estudios que evalúan esta relación y sobretodo en este grupo etario.

\section{Material y Método}

Estudio observacional transversal en una población de estudiantes de la Universidad de la Sierra Sur, realizado con el objetivo de evaluar la relación entre las piernas cortas con la presencia de SM y sus componentes. En estudiantes activos de la misma, la cual está ubicada en la población semi urbana de Miahuatlán de Porfirio Díaz Oaxaca, México; región cuya marginación es considerable y cuyo desarrollo a nivel de infraestructura y servicios aún es deficiente para considerarse una zona urbanizada. El estudio posterior a su convocatoria inició la evaluación de pacientes en la primera semana de noviembre a la segunda semana de diciembre del mismo año. Posterior a ello, se recopilaron datos, se analizaron y se tuvieron resultados en los primeros días de febrero de 2019.

El tamaño muestral se calculó sobre una población de 1063 estudiantes del segundo a décimo semestre de todas las licenciaturas, con un nivel de confianza del $95 \%$, proporción esperada de prevalencia de longitud relativa de piernas de acuerdo con un estudio realizado por Frisancho de $24.4 \%$ (Frisancho, 2007), además del 1\% de margen de error considerando las posibles bajas dentro del estudio. Con ello, se obtuvo una muestra de 224 estudiantes, mismos que fueron seleccionados por muestreo aleatorio simple, mediante un padrón de listas de asistencia proporcionadas por el área de servicios escolares de la universidad.

En los criterios de selección se consideraron a hombres y mujeres reconocidos como alumnos vigentes en la universidad, con edades entre 18 a 30 años y que estuvieran dispuestos a participar, mientras que los criterios de exclusión fueron embarazo e incapacidad para mantener una posición de pie y erguida, el único criterio de eliminación fue la nula disposición en la participación.

Para determinar que fueran alumnos activos se solicitó información a servicios escolares y a directores de cada licenciatura en la universidad sobre el estado académico de cada alumno. Posterior a convocarlos y autorizando su participación por el consentimiento informado, se aplicó una encuesta consultando datos como la edad, estado de embarazo y se hizo observación visual de los alumnos para determinar que no tuvieran dificultad para colocarse de pie o sufrieran de una discapacidad para ello. 
El estudio constó de tres fases, las cuales se describen a continuación:

Fase de reclutamiento. Una vez identificados a los candidatos para el estudio, se convocó a una reunión informativa donde se especificaron los objetivos de la investigación, las garantías y responsabilidades de cada sujeto al acceder a esta y se proporcionó un consentimiento informado. Aquellos que decidieron participar se les programaron citas para evaluarlos solicitando vinieran en condiciones idóneas para evitar sesgos en la evaluación.

Fase de evaluación. Se realizaron las siguientes mediciones: peso, talla, talla sentado, circunferencia de cintura y cadera, lo anterior de acuerdo con los protocolos propuestos por Frisancho, los criterios de referencia para la talla sentado se establecieron por este mismo (Frisancho, 2008), los del Índice de Masa Corporal (IMC) por la Organización Mundial de la Salud (OMS, 2019). Como parte de la evaluación clínica y bioquímica se midió la tensión arterial de acuerdo con el American College of Cardiology/American Heart Association Task Force (2017), y la glucosa en ayuno respectivamente, con base a la Guía de Práctica Clínica para el Tratamiento de la Diabetes Mellitus Tipo II (2018).

Fase diagnóstica. De acuerdo con los resultados obtenidos en la medición de longitud relativa de pierna, se seleccionaron los casos considerados fuera de la normalidad que de acuerdo con Frisancho se encontrarán en percentiles debajo de 5 o arriba de 95 y se les dio una nueva cita. Durante esta fase, se agregó la determinación del perfil de lípidos (triglicéridos y colesterol), los elementos evaluados se midieron a partir fotometría de reflexión. La interpretación de los valores se realizó con base en los puntos de corte de normalidad propuestos por el ATP III. El objetivo de esta fase en conjunto con el análisis estadístico permitió determinar la asociación entre la longitud relativa de pierna, el síndrome metabólico y sus componentes.

Para evitar sesgo en la evaluación de criterios antropométricos, solo un investigador se encargó de las mediciones antropométricas, mientras que alguien más realizó las clínicas y otra las bioquímicas. Aunque todos los integrantes del equipo de evaluación tuvieron una capacitación previa para estandarizar mediciones y estuvieran claros los objetivos del estudio.

A los alumnos se les solicitó vinieran en condiciones para la evaluación como vestir ropa ligera y cómoda para la toma de peso, no haber realizado actividad física extenuante en 24 horas antes de la evaluación ya que podría alterar los resultados a nivel de la composición corporal, así como un ayuno no mayor a 8 horas, evitar el consumo de alcohol y cafeína para que no modificará las mediciones clínicas y bioquímicas.

De un universo de 1063 estudiantes se seleccionaron 224, en el proceso ningún estudiante rechazo la invitación, solamente se eliminó a dos sujetos que al momento de la evaluación antropométrica no pudieron mantener una posición de pie y erguida.

Los datos fueron ingresados al software estadístico SPSS versión 22, realizándose pruebas de normalidad de acuerdo con la prueba de Kolmogorov Smirnov (corrección de significación de lilliefors), con un grado de libertad de 224, encontrándose un valor de significancia por encima de 0.05 , con lo que se asume una distribución normal. Los datos se analizaron con pruebas paramétricas. Conforme a los objetivos de este estudio se estimaron las prevalencias de LRP cortas y largas, una vez teniendo los datos se realizó la división por sexo y se obtuvo la frecuencia de SM y sus componentes en estos grupos. Para determinar la asociación entre piernas cortas/largas, SM y sus componentes se usó el método de comparación de grupos extremos o también denominado grupos conocidos, el cual consta en formar dos grupos: uno por sujetos que poseen el atributo medible en alto grado (piernas largas) y otro en donde los sujetos lo presenten en grado mínimo (piernas cortas). No se consideró a sujetos con piernas en normalidad, porque no presentan ninguno de los criterios establecidos para este análisis.

Se realizó el análisis de comparación múltiple de medias entre hombres y mujeres con piernas cortas y cada uno de los componentes clínicos, bioquímicos y antropométricos del síndrome metabólico Para determinar significancia estadística se utilizó regresión logística binaria, considerando un valor de $\mathrm{p} \leq 0.05$ 


\section{Resultados}

La población de 224 estudiantes se integró de mujeres en un $71 \%$ y hombres en $29 \%$, con una media de edad de $21.07 \pm 2.20$ años, con datos basales a nivel antropométrico, bioquímico y clínico que pueden apreciarse en la Tabla 1.

\begin{tabular}{|c|c|c|}
\hline & Hombres & Mujeres \\
\hline Variables Antropométricas & Media \pm DS & Media \pm DS \\
\hline Talla (cm) & $167.10 \pm 6.01$ & $155.44 \pm 5.33$ \\
\hline Talla sentado $(\mathrm{cm})$ & $88.54 \pm 3.12$ & $82.82 \pm 2.72$ \\
\hline $\operatorname{LRP}(\mathrm{cm})$ & $46.99 \pm 1.13$ & $46.69 \pm 1.27$ \\
\hline Peso (kg) & $67.32 \pm 12.01$ & $56.97 \pm 9.10$ \\
\hline Circunferencia de cintura (cm) & $80.92 \pm 8.50$ & $74.97 \pm 7.81$ \\
\hline Circunferencia de cadera $(\mathrm{cm})$ & $94.66 \pm 7.89$ & $93.91 \pm 6.88$ \\
\hline Índice de Masa Corporal-IMC (kg/m²) & $23.64 \pm 5.10$ & $23.55 \pm 3.40$ \\
\hline \multicolumn{3}{|l|}{ Bioquímicas } \\
\hline Glucosa en ayuno (mg/dl) & $101.55 \pm 13.70$ & $101.28 \pm 13.60$ \\
\hline \multicolumn{3}{|l|}{ Clínicas } \\
\hline Tensión arterial sistólica (mmHg) & $116.38 \pm 10.48$ & $105.98 \pm 12.15$ \\
\hline Tensión arterial diastólica $(\mathrm{mmHg})$ & $69.61 \pm 9.98$ & $64.43 \pm 8.98$ \\
\hline
\end{tabular}

Prevalencia del retraso en el crecimiento en la población universitaria

La prevalencia de piernas cortas en la población general fue de $10.7 \%$, sin diferencias por sexo (mujeres $\mathrm{p}=0.196$ y hombres $\mathrm{p}=0.125$ ) y únicamente en las mujeres se encontraron casos de piernas largas en un $0.9 \%$ del total, como puede observarse en la Tabla 2.

\begin{tabular}{|c|c|c|c|}
\hline $\begin{array}{l}\text { Distribución por } \\
\text { sexo }\end{array}$ & $\begin{array}{c}\text { LRP }<\text { percentil } 5 \\
\text { (piernas cortas) }\end{array}$ & $\begin{array}{c}\text { LRP percentil 5-95 } \\
\text { (piernas longitud normal) }\end{array}$ & $\begin{array}{c}\text { LRP> percentil } 95 \\
\text { (piernas largas) }\end{array}$ \\
\hline Femenino & $8.4 \%(5.1 \%-13.7 \%)$ & $90.4 \%(84.9 \%-93.9 \%)$ & $1.2 \%(0.3 \%-4.3 \%)$ \\
\hline Masculino & $10.9 \%(4.9 \%-21.1 \%)$ & $89.5 \%(78.9 \%-95.1 \%)$ & $0.00 \%$ \\
\hline Población total & $8.9 \%(5.9 \%-13.4 \%)$ & $90.2 \%(85.6 \%-93.4 \%)$ & $0.9 \%(0.3 \%-3.2 \%)$ \\
\hline
\end{tabular}

Prevalencia de síndrome metabólico en jóvenes universitarios con piernas cortas

Con base a los criterios diagnósticos del ATP III, en las mujeres y hombres con piernas cortas la prevalencia fue de $40 \%$ y $25 \%$ respectivamente. Hubo casos de mujeres con piernas largas sin embargo no cumplieron con los criterios suficientes para ser diagnosticadas con SM. En hombres no hubo casos de piernas largas.

Prevalencia de componentes en sujetos con síndrome metabólico

En la Figura 1 se puede apreciar que las mujeres con piernas cortas presentaron un mayor número de componentes del SM.

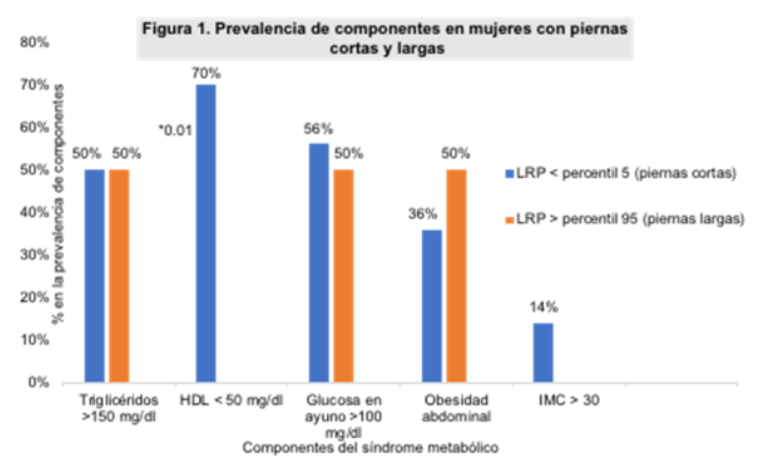

Mientras tanto en los hombres con piernas cortas se encontró una mayor presencia de componentes como triglicéridos en un 33\%, colesterol HDL 33\% y glucosa $16 \%$. Tanto hombres y mujeres presentaron valores normales de tensión arterial.

Asociación entre la longitud relativa de pierna corta y los componentes del SM

Se realizó el análisis de comparaciones múltiples de medias en donde se compararon las piernas cortas (percentil<5) y piernas largas (percentil>95) con cada uno de los componentes clínicos, bioquímicos y antropométricos del SM, de acuerdo con ello no se encontró significancia estadística en ninguno de los casos (Ver Tabla 3). 
Tabla 3. Comparaciones múltiples

\begin{tabular}{|c|c|c|c|c|c|c|}
\hline \multirow{2}{*}{$\begin{array}{c}\text { Variable } \\
\text { dependiente }\end{array}$} & \multirow{2}{*}{$\begin{array}{l}\text { (I)piernas cortas, } \\
\text { normales y largas }\end{array}$} & \multirow{2}{*}{$\begin{array}{l}\text { (J) piernas cortas, } \\
\text { normales y largas }\end{array}$} & \multirow{2}{*}{$\begin{array}{c}\text { Diferencia de } \\
\text { medias (I-J) }\end{array}$} & \multirow{2}{*}{$\begin{array}{c}\text { Error } \\
\text { estándar }\end{array}$} & \multicolumn{2}{|c|}{ Intervalo de confianza al 95\% } \\
\hline & & & & & Límite inferior & Límite superior \\
\hline \multirow{6}{*}{$\begin{array}{l}\text { Tensión arterial } \\
\text { sistólica(mm/hg) }\end{array}$} & \multirow{2}{*}{ piernas cortas } & piernas normales & 1.9078 & 2.7257 & -4.668 & 8.483 \\
\hline & & piernas largas & -2.7083 & 9.2813 & -25.098 & 19.681 \\
\hline & \multirow{2}{*}{ piernas normales } & piernas cortas & -1.9078 & 2.7257 & -8.483 & 4.668 \\
\hline & & piernas largas & -4.6162 & 8.9621 & -26.236 & 17.003 \\
\hline & \multirow{2}{*}{ piernas largas } & piernas cortas & 2.7083 & 9.2813 & -19.681 & 25.098 \\
\hline & & piernas normales & 4.6162 & 8.9621 & -17.003 & 26.236 \\
\hline \multirow{6}{*}{$\begin{array}{c}\text { Tensión arterial } \\
\text { Diastólica(mm/hg) }\end{array}$} & \multirow{2}{*}{ piernas cortas } & piernas normales & 1.7146 & 2.0575 & -3.249 & 6.678 \\
\hline & & piernas largas & -2.25 & 7.006 & -19.151 & 14.651 \\
\hline & \multirow{2}{*}{ piernas normales } & piernas cortas & -1.7146 & 2.0575 & -6.678 & 3.249 \\
\hline & & piernas largas & -3.9646 & 6.7651 & -20.284 & 12.355 \\
\hline & \multirow{2}{*}{ piernas largas } & piernas cortas & 2.25 & 7.006 & -14.651 & 19.151 \\
\hline & & piernas normales & 3.9646 & 6.7651 & -12.355 & 20.284 \\
\hline \multirow{6}{*}{ Glucosa $(\mathrm{mg} / \mathrm{dl})$} & \multirow{2}{*}{ piernas cortas } & piernas normales & 2.168 & 2.96 & -4.97 & 9.31 \\
\hline & & piernas largas & 4.25 & 10.067 & -20.04 & 28.54 \\
\hline & \multirow{2}{*}{ piernas normales } & piernas cortas & -2.168 & 2.96 & -9.31 & 4.97 \\
\hline & & piernas largas & 2.082 & 9.722 & -21.37 & 25.54 \\
\hline & \multirow{2}{*}{ piernas largas } & piernas cortas & -4.25 & 10.067 & -28.54 & 20.04 \\
\hline & & piernas normales & -2.082 & 9.722 & -25.54 & 21.37 \\
\hline \multirow{6}{*}{$\%$ Grasa } & \multirow{2}{*}{ piernas cortas } & piernas normales & -2.4907 & 1.6426 & -6.453 & 1.472 \\
\hline & & piernas largas & -9.8917 & 5.5933 & -23.385 & 3.601 \\
\hline & \multirow{2}{*}{ piernas normales } & piernas cortas & 2.4907 & 1.6426 & -1.472 & 6.453 \\
\hline & & piernas largas & -7.401 & 5.4009 & -20.43 & 5.628 \\
\hline & \multirow{2}{*}{ piernas largas } & piernas cortas & 9.8917 & 5.5933 & -3.601 & 23.385 \\
\hline & & piernas normales & 7.401 & 5.4009 & -5.628 & 20.43 \\
\hline \multirow{6}{*}{ Grasa (kg) } & \multirow{2}{*}{ piernas cortas } & piernas normales & -0.95046 & 1.38113 & -4.2822 & 2.3813 \\
\hline & & piernas largas & -6.48295 & 4.70288 & -17.8279 & 4.862 \\
\hline & piernas normales & piernas cortas & 0.95046 & 1.38113 & -2.3813 & 4.2822 \\
\hline & & piernas largas & -5.5325 & 4.54114 & -16.4873 & 5.4223 \\
\hline & piernas largas & piernas cortas & 6.48295 & 4.70288 & -4.862 & 17.8279 \\
\hline & קrentas iaryas & piernas normales & 5.5325 & 4.54114 & -5.4223 & 16.4873 \\
\hline & piernas cortas & piernas normales & 2.19667 & 1.7253 & -1.9653 & 6.3587 \\
\hline & pientas coutas & piernas largas & 3.62462 & 5.87479 & -10.5474 & 17.7966 \\
\hline Masa libre de & piernas normales & piernas cortas & -2.19667 & 1.7253 & -6.3587 & 1.9653 \\
\hline & & piernas largas & 1.42795 & 5.67275 & -12.2567 & 15.1126 \\
\hline & niernas largas & piernas cortas & -3.62462 & 5.87479 & -17.7966 & 10.5474 \\
\hline & 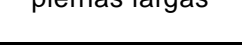 & piernas normales & -1.42795 & 5.67275 & -15.1126 & 12.2567 \\
\hline & piernas cortas & piernas normales & 1.7091 & 1.816 & -2.672 & 6.09 \\
\hline & pientas cutias & piernas largas & -1.6667 & 6.1836 & -16.584 & 13.25 \\
\hline Circunferencia de & piernas normale & piernas cortas & -1.7091 & 1.816 & -6.09 & 2.672 \\
\hline clntura (cm) & pientas mintiates & piernas largas & -3.3758 & 5.9709 & -17.78 & 11.028 \\
\hline & & piernas cortas & 1.6667 & 6.1836 & -13.25 & 16.584 \\
\hline & plerr & piernas normales & 3.3758 & 5.9709 & -11.028 & 17.78 \\
\hline
\end{tabular}

Fuente: Elaboración propia. 


\section{Artículo Original}

Asimismo, al realizar el análisis de regresión logística binaria no se encontró asociación significativa entre la longitud relativa de piernas cortas en ambos sexos con los componentes del síndrome metabólico, como puede verse en la Tabla 4. Por lo cual se acepta la hipótesis nula de la investigación.

Tabla 4. Modelo de regresión lineal múltiple

\begin{tabular}{lccr}
\hline \multicolumn{1}{c}{ Variables } & B & IC del 95\% & \multicolumn{1}{c}{ DE } \\
\hline Glucosa en ayuno $(\mathrm{mg} / \mathrm{dl})$ & -0.007 & -.055 a .042 & 17.15 \\
Circunferencia de cintura $(\mathrm{cm})$ & -0.092 & -.197 a .014 & 12.08 \\
Tensión arterial sistólica $(\mathrm{mm} / \mathrm{hg})$ & 0.101 & -.044 a .247 & 10.03 \\
Tensión arterial diastólica $(\mathrm{mm} / \mathrm{hg})$ & 0.012 & -.141 a .165 & 8.04 \\
Triglicéridos $(\mathrm{mg} / \mathrm{dl})$ & 0.002 & -.003 a .006 & 200.27 \\
Colesterol-HDL $(\mathrm{mg} / \mathrm{dl})$ & -0.008 & -.073 a .057 & 13.65 \\
\hline
\end{tabular}

Variable dependiente: LRP. IC: Intervalo de confianza

Fuente: Elaboración propia.

\section{Discusión}

Para esta investigación se evaluó la proporción corporal a partir de la toma de LRP propuesta por Frisancho, por cuestiones de uniformidad (sexo, edad y nacionalidad) y al ser la más utilizada en otros estudios (Frisancho, 2007). Encontrando presencia de longitudes bajas en un $10.7 \%$, de los cuales $32.5 \%$ tenía SM y de esta manera no observándose relación entre las piernas cortas como factor a la presencia de SM ni de las piernas largas como prevención al mismo. Dentro de las limitaciones del presente, se encuentra la heterogenicidad por sexos, dado a que el mayor número estudiantil en la población evaluada es de mujeres observándose esto mismo en los resultados y que si se hubiese evaluado algunas características socioeconómicas y geográficas se hubiera tenido un análisis más enriquecedor.

La prevalencia de LRP cortas fue muy similar en comparación con otros estudios donde se evaluó a población mexicana, como el estudio en mujeres migrantes con un $12 \%$ por parte de Vilar-Compte y cols. y el estudio de Malina y cols. en población adolescente en el estado de Oaxaca con un 19\% (Vilar-Compte et al., 2019; Malina et al., 2004). De acuerdo con las estadísticas nacionales, se esperaba encontrar mayor número de pacientes con piernas cortas, tomando en consideración que la región sur del país tiene tres veces más probabilidades de presentar población con estaturas bajas $(40 \%)$ en comparación de la región norte (12.2\%) (CastroPorras et al., 2018) sin embargo, la ENSANUT también ha demostrado tener un descenso de la talla baja en 12.7 puntos porcentuales (pp) entre 1988 a 2012 y se estima este descenso en 0.55 pp/año (ENSANUT, 2018).

Estudios como el de Malina y cols. en Oaxaca y el de Padez y cols. en población Mozambique indican que a pesar de las situaciones adversas que viven los países subdesarrollados, es la transición de localidades rurales a urbanas, el mestizaje y los cambios en los patrones alimenticios algunas de las condicionantes por las que la prevalencia de estaturas disminuyera en regiones donde las estaturas bajas eran características (Malina et al., 2004; Padez, Varela-Silva y Bogin, 2009).

Otro resultado importante fue la prevalencia de síndrome metabólico en la población de estudio la cual fue del $32.5 \%$, cifra similar al estudio elaborado en Oaxaca en 2007 por Ramírez-Vargas y cols. que evaluaron la presencia de SM en comunidades rurales y urbanas de este estado ( $27.6 \%$ vs $45.4 \%$ ), dicha prevalencia era esperada considerando que Miahuatlán es un municipio semi urbano que guarda diversas costumbres símiles a las comunidades rurales (Ramírez-Vargas, Arnaud y Delisle, 2007).

En el estudio no se encontró asociación entre los componentes de SM y la LRP corta, a diferencia de otros estudios (Briskiewicz et al., 2018; Liu et al., 2014), probablemente por cuestiones metodológicas como un universo mayor en los mismos, la distribución equitativa por género y poblaciones en una adultez mayor donde hay probabilidad de encontrar indicadores antropométricos y bioquímicos fuera de los rangos de normalidad. En este estudio como el de Malina y cols., de igual forma no se encontró asociación de la LRP y el SM, al considerarse la edad uno de los factores protectores ante el mismo (Malina et al., 2004).

El estudio tiene distintas áreas de oportunidad, pero también se considera importante al ser de los pocos con la intención de demostrar la asociación entre la proporción corporal y el SM, como recomendaciones para futuros investigadores se sugiere tener una muestra poblacional mayor, distribuida equitativamente por género y edad, además de estudiar variables socioeconómicas y ambientales que aseguren mejor predicción a la presencia de LRP cortas. 


\section{Conclusiones}

El SM y sus componentes se han convertido en prioridades de la salud pública a nivel nacional y mundial, sin embargo, su diagnóstico y su predicción sobre el riesgo a presentarlo aún siguen siendo relevantes. Esta investigación se centró en la importancia que tiene la estatura y la distribución de segmentos corporales en el riesgo a presentar SM.

Se encontró que en la población de estudio $10.7 \%$ tuvieron piernas cortas y de estos $32.5 \%$ tuvieron $\mathrm{SM}$, sin embargo, al hacer en análisis de correlación no se encontró significancia estadística. Por ende, se considera que para este estudio la LRP corta no es un factor a la presencia de SM y sus componentes.

Es importante destacar la relevancia del estudio, al ser de los pocos que evalúa la distribución corporal y su asociación con el SM en el país y sobretodo en población joven. Además de que es importante sumar estrategias de promoción a la salud con el fin de evitar complicaciones cardio-metabólicas más severas.

\section{Bibliografía}

Aguilar-Salinas, C. A. y Rojas, R. (2012). Epidemiología de la diabetes y el síndrome metabólico en México. Ciencia, 36-4.

Bogin, B. y Varela, M. (2010). Leg light, body proportion, and health: a review with a note on beauty. International journal of environmental research and public, 7 (10), 1047- 1075.

Briskiewicz, B. L., Barreto, S. M., Do Amaral, J., Diniz, M., Molina, M., Matos, S., Cardoso, L., Velasquez-Melendez, G., Schmidt, M. y Giatti, L. (2018). Early-life nutritional status and metabolic syndrome: Gender-specific associations from a cross-sectional analysis of the Brazilian Longitudinal Study of Adult Health (ELSA-Brasil). Public health nutrition, 21 (8), 1546-1553

Castro-Porras, L. V., Rojas-Rusell, M.E., AedoSantos, Á., Wynne-Bannister, E.G. y LópezCervantes, M. (2018). Stature in adults as an indicator of socioeconomic inequalities in Mexico. Revista Panamericana de Salud Pública, 42 (1).
Diagnóstico y Tratamiento Farmacológico de la Diabetes Mellitus Tipo 2 en el Primer Nivel de Atención. Guía de Evidencias y Recomendaciones: Guía de Práctica Clínica. México, Instituto Mexicano del Seguro Social; 2018

Encuesta Nacional de Salud y Nutrición (ENSANUT, 2018). Instituto Nacional de Estadística y Geografía INEGI [Internet]. Disponible en: https://ensanut.insp.mx/encuestas/ensanut2018/d octos/informes/ensanut_2018_pres entacion_resultados.pdf

Fischer, L. y Galler, J. (2015). Early childhood malnutrition increases metabolic syndrome in adulthood. Federation of American societies for de experimental biology, 2 (1), 258-2.

Frisancho, A. R. (2007). Relative leg length as a biological marker to trace the developmental history of individuals and populations: Growth delay and increased body fat. American journal of human biology, 19 (5), 703-710.

Frisancho, A. R. (2008). Anthropometric standards: an interactive nutritional reference of body size and body composition for children and adults. Michigan: University of Michigan Press.

Guideline for the prevention, detection, evaluation, and management of high blood pressure in adults. New York: American College of Cardiology Foundation and the American Heart Association, Inc.; 2017.

Hales, C. y Barker, D. (2013). Type 2 (non-insulindependent) diabetes mellitus: the thrifty phenotype hypothesis. International journal of epidemiology, 42 (5), 1215-1222.

Lawlor, D. A., Taylor, M., Davey, G., Gunnell, D. y Ebrahim, S. (2004). Associations of components of adult height with coronary heart disease in postmenopausal women: The British women's heart and health study. Heart, 90 (7), 745-749.

Malina, R. M., Peña, M. E., Tan, S. K., Buschang, P. H., Little, B. y Koziel, S. (2004). Secular change in height, sitting height and leg length in rural 
Oaxaca, Southern Mexico: 1968-200. Annals of human biology,31 (6), 615-633.

Mueller, N.T. y Pereira, M. A. (2015). Leg length and type 2 diabetes: what's the link? Current opinion in clinical nutrition and metabolic care, $18(5), 452-456$.

Organización Mundial de Salud. (16 de febrero de 2019). Obesidad y sobrepeso. Recuperado de https://www.who.int/es/news-room/factsheets/detail/obesity-and-overweight

Padez, C., Varela-Silva, M. I. y Bogin, B. (2009). Height and relative leg length as indicators of the quality of the environment among Mozambican juveniles and adolescents. American journal of human biology: the official journal of the Human Biology Council, 21(2), 200-209.

Ramírez-Vargas, E., Arnau, M. y Delisle, H. (2007). Prevalence of the metabolic syndrome and associated lifestyles in adult males from Oaxaca, Mexico. Salud Pública de México, 49 (2), 94102.

Sawaya, A. L., Martins, P., Hoffman, D. y Roberts, S. B. (2003). The link between childhood undernutrition and risk of chronic disease in adulthood: a case study of Brazil. Nutrition, 61 (1), 168-175.)

Sawaya, A. L. y Roberts, S. (2003). Stunting and future risk of obesity: principal physiological mechanisms. Cadernos de Saúde Pública, 19 (1), 21-28.

Vilar-Compte, M., Jacinko, J., Weitzman, B. y Avendaño, C. (2019). Short relative leg length is associated with overweight and obesity in Mexican immigrant women. International journal for equity in health, 18(1), 103. 